Article

\title{
Surrogate Based Optimization of Aerodynamic Noise for Streamlined Shape of High Speed Trains
}

\author{
Zhenxu Sun *, Ye Zhang and Guowei Yang \\ Key Laboratory for Mechanics in Fluid Solid Coupling Systems, Institute of Mechanics, Chinese Academy of \\ Sciences, Beijing 100190, China; zhangye@imech.ac.cn (Y.Z.); gwyang@imech.ac.cn (G.Y.) \\ * Correspondence: sunzhenxu@imech.ac.cn; Tel.: +86-10-8254-3815
}

Academic Editors: Roberto Citarella and Luigi Federico

Received: 3 January 2017; Accepted: 13 February 2017; Published: 17 February 2017

\begin{abstract}
Aerodynamic noise increases with the sixth power of the running speed. As the speed increases, aerodynamic noise becomes predominant and begins to be the main noise source at a certain high speed. As a result, aerodynamic noise has to be focused on when designing new high-speed trains. In order to perform the aerodynamic noise optimization, the equivalent continuous sound pressure level (SPL) has been used in the present paper, which could take all of the far field observation probes into consideration. The Non-Linear Acoustics Solver (NLAS) approach has been utilized for acoustic calculation. With the use of Kriging surrogate model, a multi-objective optimization of the streamlined shape of high-speed trains has been performed, which takes the noise level in the far field and the drag of the whole train as the objectives. To efficiently construct the Kriging model, the cross validation approach has been adopted. Optimization results reveal that both the equivalent continuous sound pressure level and the drag of the whole train are reduced in a certain extent.
\end{abstract}

Keywords: aerodynamic noise; NLAS; kriging model; cross validation; equivalent continuous SPL

\section{Introduction}

As the running speed of high-speed trains increases, aerodynamic noise becomes to be predominant and takes the major part of train noise. Severe noise problem will cause discomfort for the passengers and surrounding people. Furthermore, it will also cause damage to surrounding equipment and buildings. Noise issue becomes a key factor that limits the increase of running speed of high-speed trains, which needs to pay enough attention in the design of new trains [1].

Current study on aerodynamic noise of high-speed trains mainly focuses on two aspects: On the one hand, identification of noise sources of high-speed trains and mechanism analysis [2-6] were performed, aiming at obtaining more precise acoustic structures of high-speed trains. Talotte presents a critical survey of the identification and modeling of railway noise sources and summarizes the current knowledge of the physical source phenomena [2,3]. Sun numerically analyzes the main noise sources of high-speed trains, quantitatively compares the different noise sources and identifies the main noise sources, such as the leading streamline, the inter-coach spacing, the bogie zone and the wake zone as well $[4,6]$. Noger performed an investigation on acoustic production mechanisms in the TGV (which is short from the French words "train à grande vitesse") pantograph recess to be able to reduce the radiated noise [5]. Until now, the study on aerodynamic noise mechanism has gradually matured. On the other hand, many studies focus on noise reduction of specific components to meet practical usage [6-10]. In order to obtain low noise pantograph, Ikada proposes a precise rail pantograph design method that provides both aerodynamic and aero-acoustic sound designs more quickly and economically $[7,8]$. West Japan Railway Co. made an investigation to find an optimum shape of the front bulkhead of the pantograph cover and at the same time actively pursues development of a wing-shaped pantograph which causes less aerodynamic noise. Iwamoto and Higashi made a 
technical view from results of their tests using the actual railcar and the wind test tunnel [9]. Sueki proposed a new aerodynamic noise reduction method that involves covering the surface of objects with a particular porous material [10]. Sun investigated on different designs of cab windows and cowcatchers and gave a reasonable advice on low noise design of the streamlined shape [6]. It can be seen that much effort has been put to the design of low-noise pantograph, which engineering experience is mostly dependent on for noise reduction. This kind of design is usually constrained by engineering experience and cannot obtain the most optimal shape. In addition, study on the noise reduction of the streamlined head of high-speed trains could be seldom referred to, since abundant flow phenomena exist on the surface of the streamline and the design approach which relies solely on engineering experience could not meet the accuracy requirement. Consequently, optimization design with corresponding algorithms is the only way to obtain the relationships between design variables and aerodynamic noise and gets the chance to obtain the optimal design.

In recent years, optimization algorithms have been widely used in the streamlined shape design of high-speed trains [11-16]. Lee and Kim dealt with the nose shape design of high-speed railways to minimize the maximum micro-pressure wave and suggested an optimal nose shape that is an improvement over current design in terms of micro-pressure wave [11]. Vytla used the Kriging model to find the optimum shape of a two-dimensional nose shape of a high-speed train traveling at $350 \mathrm{~km} / \mathrm{h}$ considering both the induced aerodynamic drag and the generated aerodynamic noise [12]. $\mathrm{Ku}$ used the VMF parameterization method on the train nose to reduce a micro-pressure wave and an aerodynamic drag of a high-speed train [13]. Yao performed a constrained multi-objective optimization design on a simplified CRH380A high-speed train with three carriages to optimize aerodynamic drag of the total train and aerodynamic lift of the trailing car and got agreeable optimal results [14]. Suzuki and Nakade presented a novel design technique for high-speed trains using a multi-objective optimization method to balance plural aerodynamic properties [15]. Muñoz-Paniagua used genetic algorithms to optimize the nose shape of a high-speed train entering a tunnel in term of the compression wave generated at the entry of the train and the aerodynamic drag of the train [16]. It could be concluded that the optimization objectives are mostly focused on the aerodynamic loads or tunnel effects. Even aerodynamic noise is considered, it is only a two-dimensional study. Due to the massive cost of computational aero-acoustics (CAA), aerodynamic noise optimization always seems untouchable for researchers. In order to perform aerodynamic noise optimization, two problems have to be solved in advance: On the one hand, the efficiency of CAA calculation has to be improved, which means proper CAA algorithm should be determined to adapt the computing scale for high-speed trains; On the other hand, assessment of the noise level in the far field should be determined. Once the above problems have been solved, the aerodynamic noise optimization becomes feasible. It is very crucial to construct an aerodynamic noise optimization strategy for practical streamlined shape of high-speed trains.

In the present paper, the authors present an aerodynamic noise optimization strategy for practical streamlined shape of high-speed trains. The NLAS approach [17] has been adopted to perform CAA analysis for each design sample. Meanwhile, the equivalent continuous SPL has been introduced to take all of the far field observation probes into consideration and taken as one objective for multi-objective optimization. With the use of Kriging surrogate model, the aerodynamic noise optimization of the streamlined shape of high-speed trains could be performed. In the present study, the noise level in the far field and the drag of the whole train are taken as the objectives. It is mainly based on the following considerations: The aerodynamic drag of high-speed trains can be up to $75 \%$ of the total drag at the speed of $300 \mathrm{~km} / \mathrm{h}$ and the drag characteristics of the trains are directly related to the ability of energy saving and environmental protection. As a result, the aerodynamic drag reduction is very prominent and has become one of the key issues for aerodynamic shape optimization of high-speed trains. Meanwhile, aerodynamic noise increases with the sixth power of the running speed, and would be predominant over mechanical noise when the running speed is over $250 \mathrm{~km} / \mathrm{h}$. It is found that simply reducing aerodynamic drag cannot efficiently reduce the aerodynamic noise, indicating that aerodynamic noise optimization has to take aerodynamic noise as one optimization objective. 
After optimization, aerodynamic performance of the optimized shape and the prototype shape with three carriages is comparatively analyzed. Finally, sensitivity analysis has been performed, so that the nonlinear relationship between the objectives and design variables could be obtained. Current research pushes forward the cognition on how to reduce the noise level by modifying the streamlined shape of high-speed trains.

\section{Algorithms}

\subsection{Acoustic Algorithms and Validation}

Two kinds of approaches are adopted in the present work, NLAS [17] to solve the near field noise and FW-H sound propagation method to solve the far field noise, respectively. For the latter approach, an acoustic surface around the noise sources is built to record fluctuation data during NLAS calculation, which would be taken as the initial value for $\mathrm{FW}-\mathrm{H}$ propagation equation. The overall solving process is shown in Figure 1:

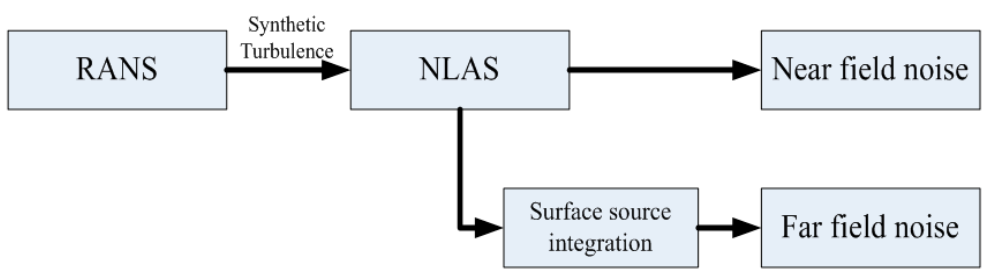

Figure 1. The schematic drawing of solution procedure for Computational aero-acoustics (CAA) calculation.

The NLAS is a numerical acoustics solver designed to model noise generation and propagation from an initial statistically-steady model of turbulent flow data, which can be provided by a simple Reynolds Averaged Navier-Stokes (RANS) turbulence model. Through the RANS calculation, the statistical steady mean flow could be obtained, from which the main generation zone of turbulence could be revealed. In the present paper, an anisotropic turbulence model, the cubic $k-\varepsilon$ model, is utilized to preferably model the statistically steady flow field [18]. Non-linear terms are taken into consideration to account for normal-stress anisotropy, swirl and streamline curvature effects. Consequently, the local Reynolds-stress tensor could be provided precisely to synthesize the noise sources. The transient calculation of NLAS should be performed on the premise of the steady RANS calculation of the flow field. NLAS uses a reconstruction procedure to generate noise sources from the given set of statistics and allows the resulting propagation of the pressure disturbances to be simulated using a high-resolution pre-conditioned solver.

The acoustic surface is used to record fluctuation data during the NLAS procedure. Once the data is obtained, Ffowcs-Williams/Hawking equation can be solved based on these data. The FW-H equation approach $[19,20]$ can be used to predict noise at any observation point outside the acoustic surface, even if the observation point is outside the computational domain. The whole CAA analysis is performed by the commercial software CAA++ (Ver 10.1, Metacomp Technologies, Los Angeles, CA, USA, 2011).

Numerical validation has already been performed in the literature by the author [4]. It took a backward step case as the test case. It comes from the experiment by Lee and Sung in a subsonic wind tunnel [21]. The flow conditions in the numerical simulation maintain the same with the experiment. In the experiment, the span wise width is 12.5 times of the height of the backward step to keep the central section two-dimensional. As a result, a two-dimensional simulation was conducted for the central section. The observation points are chosen just on the floor behind the step, as the experiment did. Fast Fourier Transformation (FFT) analysis is performed on the pressure fluctuating data. Meanwhile, numerical simulation with the Large Eddy Simulation (LES) approach 
was also performed. The results from the NLAS approach, the LES approach and the experiment are listed together for comparison, which mainly focuses on the sound pressure level and corresponding dominant frequency (Tables 1 and 2).

Table 1. Maximal sound pressure level (decibel, dB), where $x$ is the distance from the probe to the step, and $H$ is the height of the step.

\begin{tabular}{cccccc}
\hline Positions & $x / H=2$ & $x / H=4$ & $x / H=6$ & $x / H=8$ & $x / H=10$ \\
\hline experiment & -26 & -27 & -24.2 & -22.6 & -24 \\
NLAS & -26 & -23 & -24.35 & -21.7 & -23.2 \\
LES & -26.27 & -25.2 & -23.04 & -18.6 & -19.54 \\
\hline
\end{tabular}

NLAS: Nonlinear Acoustics Solver; LES: Large Eddy Simulation.

Table 2. Dominant frequency $(\mathrm{Hz})$, where $x$ is the distance from the probe to the step, and $H$ is the height of the step.

\begin{tabular}{cccccc}
\hline Positions & $\boldsymbol{x} / \mathbf{H}=\mathbf{2}$ & $\boldsymbol{x} / \mathbf{H}=\mathbf{4}$ & $\boldsymbol{x} / \mathbf{H}=\mathbf{6}$ & $\boldsymbol{x} / \mathbf{H = 8}$ & $\boldsymbol{x} / \mathbf{H}=\mathbf{1 0}$ \\
\hline experiment & 11.5 & 10.5 & 18 & 12 & 18 \\
NLAS & 14 & 7.1 & 13 & 13 & 9.1 \\
LES & 13.9 & 14.5 & 28.5 & 28.5 & 43 \\
\hline
\end{tabular}

It can be seen that better dominant frequencies are achieved through the NLAS approach, except for the position $x / H=10$. Meanwhile, the maximal sound pressure level predicted by NLAS agrees well with the experimental data. However, the maximal sound pressure level and dominant frequency obtained by LES under the same mesh and flow conditions show relative variation with experimental data. This can be related to that the synthetic reconstruction of sub-grid sources can be achieved in NLAS approach rather than LES approach, which makes the results from NLAS approach seems much more accurate. Simulation results reveal that the NLAS approach is an efficient and high-resolution computational method for aerodynamic noise prediction, and can be adopted for the large-scale computation of aerodynamic noise generated by high-speed trains.

\subsection{Assessment of the Noise Level in the Far Field}

When performing the CAA calculation, the train is assumed stationary, while the air passes by the train. Consequently, acoustic probes are placed equidistantly along the train to perform noise analysis. Due to the relatively large number of acoustic probes, it is difficult to assess the noise level by only one or two probes among them. When the acoustic optimization is conducted, it is necessary to take all the acoustic probes into consideration. Considering that the real circumstance is that the train passes by the probes one by one with the same speed, the equivalent continuous sound pressure level (SPL) is introduced in the present paper, which takes the form as:

$$
L_{A e q}=10 \lg \left[\frac{1}{T} \int_{0}^{T} 10^{0.1 L_{A}} d t\right]
$$

where $T$ is the measurement time and $L_{A}$ is the A-weighted SPL at time $t$.

As long as the noise probes are placed equidistantly, the equivalent continuous SPL could be discretized as shown below:

$$
L_{\text {Aeq }}=10 \lg \left[\frac{1}{n} \sum_{i=1}^{n} 10^{\frac{L_{A i}}{10}}\right]
$$

where $n$ is the number of the acoustic probes and $L_{A i}$ is the A-weighted SPL at the probe $i$.

Since the equivalent continuous SPL could takes all the acoustic probes in the far field into consideration, here it is taken as the objective to represent the noise level in the far field for the whole train. 


\subsection{Algorithms for Computational Fluid Dynamics (CFD) and Validation}

As one goal of the whole optimization process, the drag of the whole train has to be evaluated, which is performed by CFD analysis. CFD accuracy directly affects the construction of the surrogate model and efficiency of optimization algorithm. In this paper, the speed of high-speed train is $300 \mathrm{~km} \cdot \mathrm{h}^{-1}$. Under this condition, air compressibility has to be considered. Therefore, the steady compressible Reynolds-averaged Navier-Stokes equations [22] based on the finite volume method are used to predict the aerodynamic drag. The $k$ - $\omega$ SST model [23] is selected as the turbulence model. The standard wall functions are used near the wall so that the accuracy of the CFD results could be ensured with a limited amount of mesh.

To validate the CFD algorithms, and to obtain a reasonable mesh configuration for aerodynamic simulation of high-speed trains, the authors performed a comparison study with wind tunnel experimental results. The experiments were performed in China Aerodynamics R\&D Center. The wind tunnel model is a 1:8 scaled train model, with the bogies and inter-coach spacing included, as shown in Figure 2a. The velocity of the air flow is $60 \mathrm{~m} / \mathrm{s}$. The computational domain is the same as the tunnel walls. Due to the complex geometry, local densification of the mesh is performed, and the total number of grids is about 21.7 million. The mesh configuration is shown in Figure $2 b$.

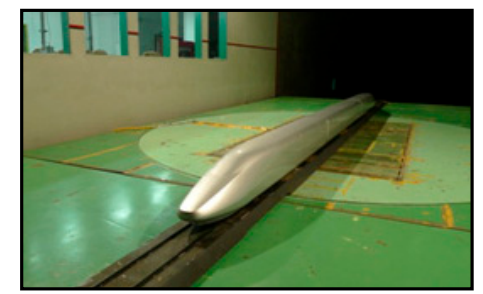

(a)

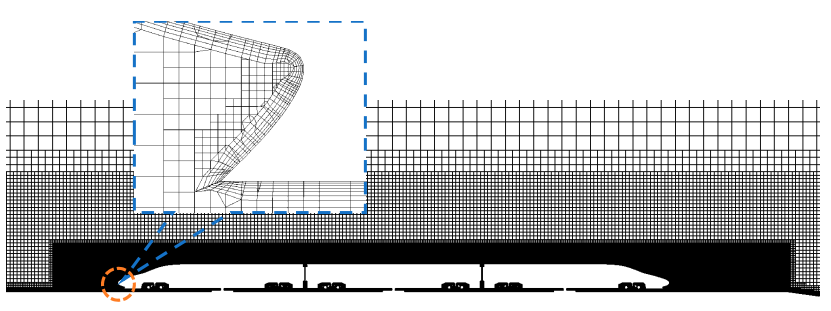

(b)

Figure 2. Wind tunnel train model and mesh configuration for numerical simulation: (a) wind tunnel model; and (b) mesh configuration.

Table 3 shows the comparison of aerodynamic coefficients between experimental results and numerical results. It can be seen that the aerodynamic coefficient error for each car are all within $5 \%$, which is acceptable for engineering application, indicating that the CFD algorithms and mesh configuration for the simulation are both feasible for aerodynamic drag evaluation of high-speed trains, and will be utilized in the present study.

Table 3. Comparison of aerodynamic coefficients between experimental results and numerical results.

\begin{tabular}{ccccc}
\hline Aerodynamic Loads & Total-Cd & Head-Cd & Middle-Cd & Tail-Cd \\
\hline Experiment & 0.326 & 0.125 & 0.082 & 0.119 \\
CFD & 0.310 & 0.118 & 0.079 & 0.113 \\
Error & $4.91 \%$ & $5.60 \%$ & $3.66 \%$ & $5.00 \%$ \\
\hline
\end{tabular}

CFD: Computational Fluid Dynamics.

\section{Local Shape Function Parametric Approach}

In this paper, a parametric approach called Local Shape Function (LSF) which is based on FFD method [24] and NURBS method [25] has been designed.

The whole processes are as follows:

(1) For a given geometry, deformation regions should be divided firstly.

(2) Mesh the deformation regions, and obtain the coordinate values of every grid point. In order to keep the smooth transition of the surface, the structural grids have been utilized for mesh discretization, as shown in Figure 3. 
(3) Choose the deformation function of each region, which can be selected randomly, but smooth transition between adjacent regions should be ensured.

(4) Choose a weight factor $W_{i}$ for each deformation function, which determines the maximum deformation value of each region.

(5) Calculate the increments $\Delta$ of coordinates of all grid points by the deformation functions and $W_{i}$.

(6) Get the coordinates of the deformed shape by summing $\Delta$ and the coordinates of the original shape.

(7) According to the coordinates of the deformed shape, the deformed surface can be fitted exactly, then a deformation process is done.

In the above process, Step (3) is the most crucial. The deformation surfaces are different from each other due to the different choices of deformation functions. Inappropriate deformation functions will easily lead to irrational deformation surfaces. Trigonometric functions, exponential functions, logarithmic functions, polynomial functions and NURBS functions are all commonly used for deformation functions.

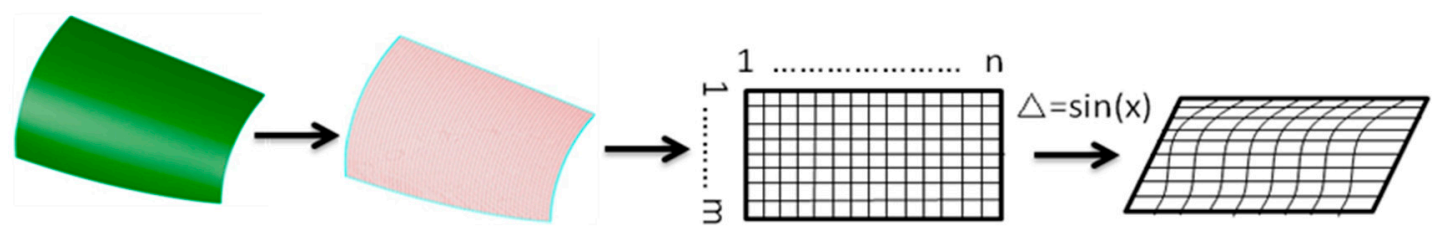

Figure 3. Schematic of surface deformation by Local Shape Function (LSF) method.

Due to the symmetrical design along the longitude of the train, only one side of the symmetrical plane of the streamline is parameterized. As a result, the design parameters can be reduced by half. The parametric surfaces are separated into four deformation regions, as shown in Figure 4a. The width of the streamline is controlled by Zone1, and a control point Point1 is set here, extracting its y coordinate as the third design parameter $w_{1}$. The slope of the cab window is controlled by Zone2, and the second control point Point 2 is set here, extracting its $\mathrm{z}$ coordinate as the fourth design parameter $w_{2}$. Nose height is controlled by Zone3, and another control point Point 3 is set here, extracting its $\mathrm{z}$ coordinate as the second design parameter $w_{3}$. Nose drainage is controlled by Zone4, and a control point Point 4 is set here, extracting its y coordinate as the first design parameter $w_{4}$. For simplicity, all the deformation functions in this paper are trigonometric functions. Figure $4 \mathrm{~b}$ shows the deformation in the height of the nose and the cab window. As seen above, the deformation method can ensure the surface smoothness and smooth transition among different deformation regions.

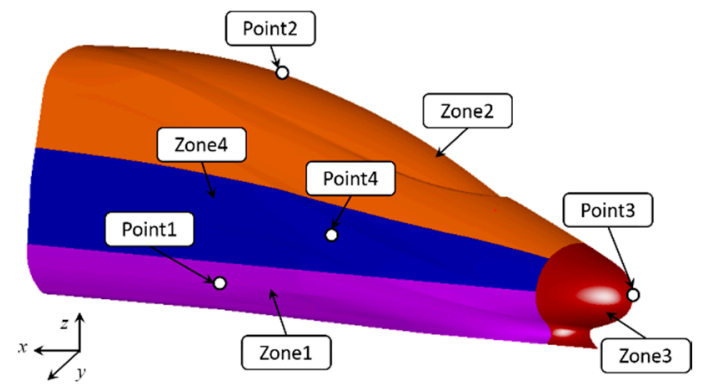

(a)

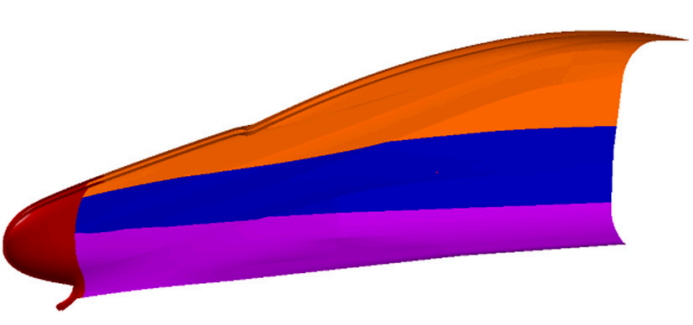

(b)

Figure 4. Deformation zones and local deformations: (a) schematic of deformation zones; and (b) the deformation of the nose and the cab window. 


\section{Optimization Strategy}

\subsection{Construction of Cross-Validation Based Kriging Model}

Considering the huge computational cost in the aerodynamic optimization, especially in the shape optimization of high-speed trains, a sequential optimization method based on minimizing the response surface criteria has been adopted in the present paper to reduce the training points. The Kriging surrogate model is an interpolation technique based on statistical theory [26-29]. This model takes full account of the relevant characteristics of the variable space, containing the regression part and the nonparametric part. The Kriging model is obtained based on the cross-validation algorithm [30]. The use of cross-validation algorithm could prolong the time of the training process. However, compared to the massive cost of CFD and CAA calculation for each sampling point, this time cost could be ignorable. A basic Kriging model could be firstly constructed from the initial 20 training points. Based on this model, the Pareto set could be obtained. Two typical points of the Pareto set could be chosen for CFD/CAA validation. If the optimization accuracy could not meet the requirement, these two points then would be added to the initial training points to build a more accurate Kriging model. If the optimization accuracy meets the design requirement, the Kriging model is finally constructed. During each iteration process, the training points are divided into groups randomly, and each group has two training points.

\subsection{Optimization Process}

Figure 5 shows the whole optimization process designed in present paper. First, the Latin hypercube method is adopted to initialize the sampling points in the design space. Then, the CFD analysis is performed to obtain the values of the objectives corresponding to the sampling points. Third, the Kriging model is constructed via genetic algorithm (GA) technique based on these sampling points. After the Kriging model has been constructed, the multi-objective optimization based on self-adaptive GA approach could be performed and the Pareto set could be obtained. Last, the final Kriging model and multi-objective self-adaptive GA approach are both adopted to obtain the Pareto solutions in the design space.

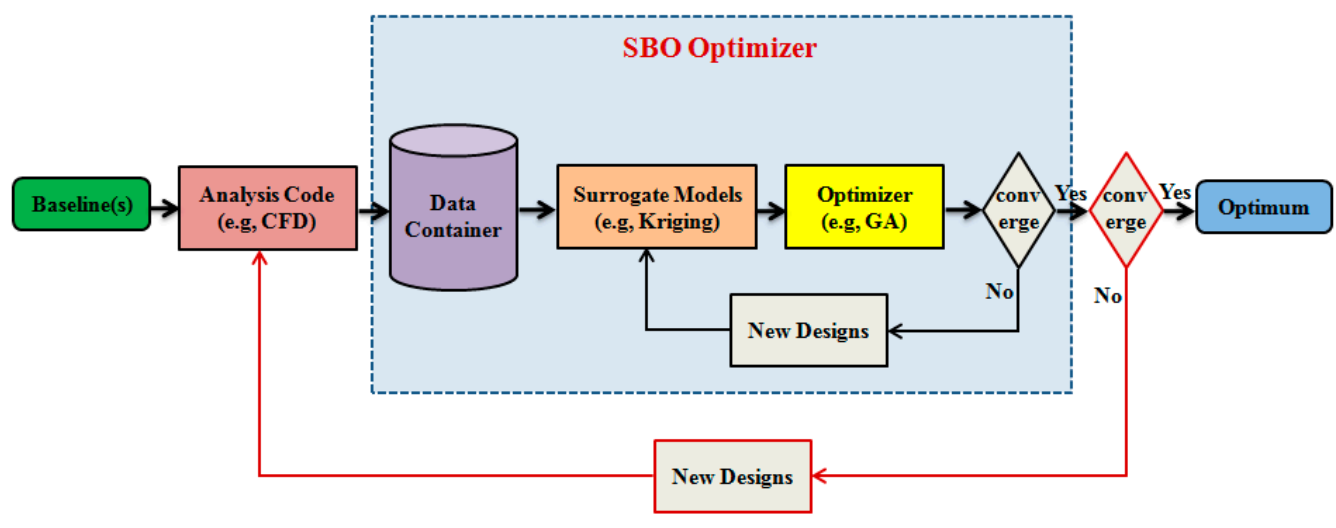

Figure 5. Schematic drawing of surrogated based optimization.

Based on the above process, multi-objective optimization of the streamlined shape of high-speed trains is performed, which will be discussed in the following sections.

\section{Computational Models, Mesh and Conditions}

A three-grouping high-speed train model has been adopted in the present paper, which is named as EMU1. The total length of the train is about $78 \mathrm{~m}$ and the height of the train is about $4.05 \mathrm{~m}$. The whole train is shown in Figure 6. 


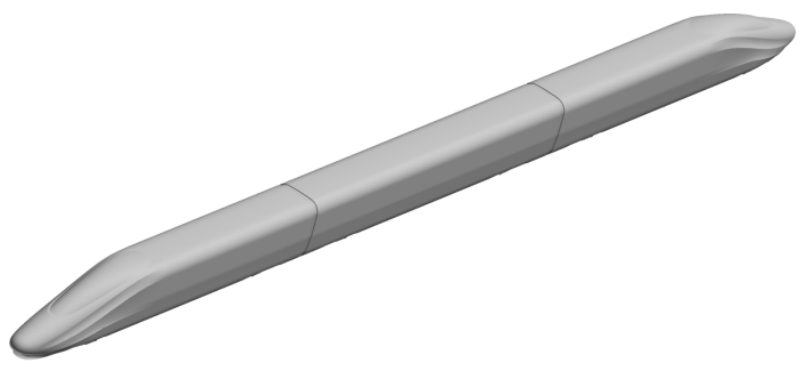

Figure 6. The whole train model.

In order to reduce the computational cost, some additional components are eliminated, such as the pantograph. When performing the CFD calculation, components such as the windshields and bogies are reserved. However, since the computational cost of CAA analysis is extremely larger than that of CFD, the windshields and bogies are both neglected to reduce the calculation time, yet the influence of the streamlined shape on acoustic characteristics could still be considered.

Take the height of the train as the characteristic length $\mathrm{H}$, the computational domain extends $30 \mathrm{H}$ ahead of the train nose and $60 \mathrm{H}$ from the train tail to the exit of the computational domain. The top of the computational domain is at a distance $30 \mathrm{H}$ from the bottom of the rail and the sides are at a distance of $30 \mathrm{H}$ from the center axis of the train, the outline of computational domain and the model are shown in Figure 7.

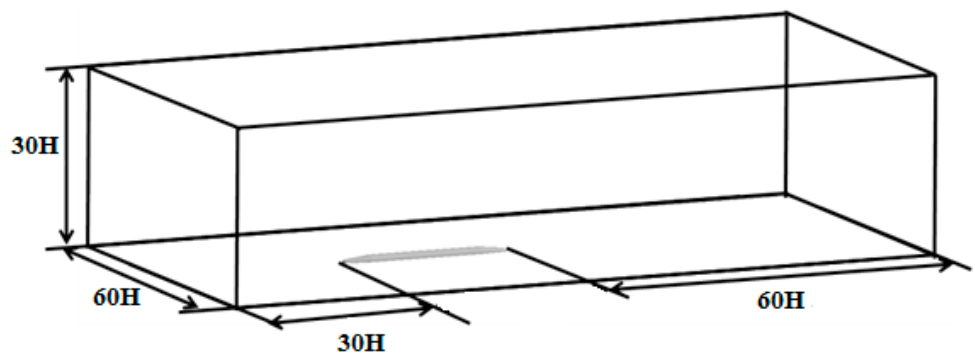

Figure 7. Computational domain.

The hybrid Cartesian/prism grids is adopted and six layers of prism grids are generated with an increasing ratio of 1.2 and a total length of $30 \mathrm{~mm}$, which keeps the value of $y+$ of the first layer near the train surface in a range of 30 100. The total number of the cells is about 32 million. Figure 8 shows the grids on the longitudinal section and on the surface of leading streamline, while Figure 9 shows the grids on the longitudinal section of wake region.

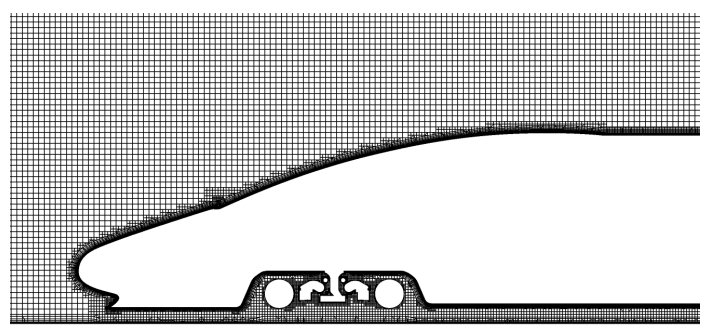

(a)

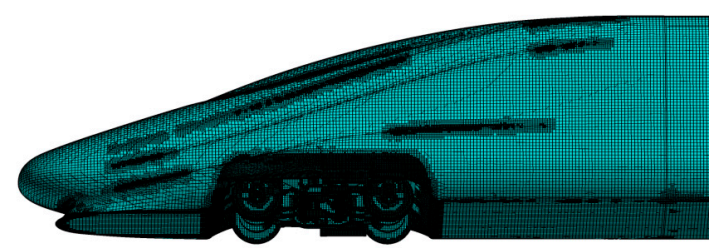

(b)

Figure 8. The grids on the longitudinal section and on the surface of leading streamline: (a) mesh distribution on the longitudinal section; and (b) surface grids of the leading streamline. 


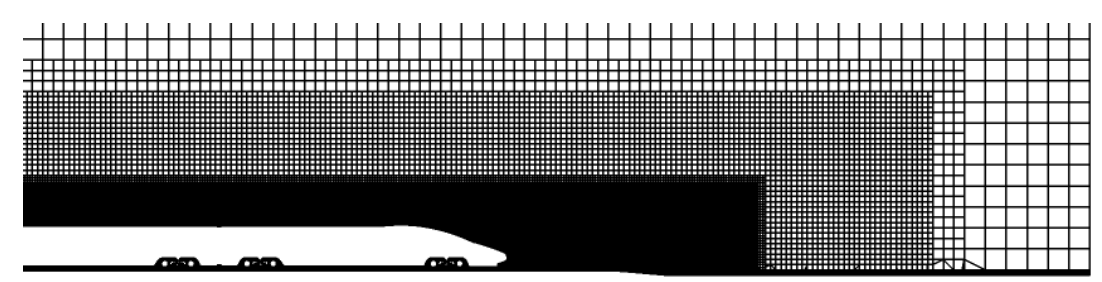

Figure 9. The grids on the longitudinal section of wake region.

The flow velocity is $300 \mathrm{~km} / \mathrm{h}$; the far-field pressure is $1 \mathrm{~atm}$; the temperature is $288 \mathrm{~K}$; and the reference area is the maximum cross-sectional area of the train. As a result of the compressibility calculation model, one-dimensional inviscid flow of the Riemann invariants is introduced as the far-field boundary conditions, which are also known as non-reflective boundary conditions. Inflow, outflow and the top boundaries are all set as far-field boundary conditions and the train body is non-slip solid wall boundary condition. The ground is treated as the moving wall to simulate the ground effect, and the moving speed is equal to the train speed.

When the NLAS procedure is conducted, three absorbing layers are imposed to the inlet boundary, the outlet boundary and the far field boundary respectively to prevent wave reflections from these boundaries. Two hundred Fourier modes are set to perform synthetic reconstruction for the turbulent fluctuating quantities to capture the sub-grid sources correctly. The time step in NLAS simulation is set to $2 \times \mathrm{e}^{-5} \mathrm{~s}$, and the simulated physical time is $0.3 \mathrm{~s}$, which insures that the noise whose frequencies locate between $10-10,000 \mathrm{~Hz}$ could be precisely predicted.

After solving the acoustic field in the near field, the far field noise could be obtained with the use of FW-H equation. The observation probes are set according to the ISO-2005-3095 standard, which requires the probes to be $25 \mathrm{~m}$ far from the train body and $3.5 \mathrm{~m}$ height from the ground. Thirteen probes are placed along the train body with an equal distance of $10 \mathrm{~m}$, which range from the leading head to the trailing streamline, as shown in Figure 10.

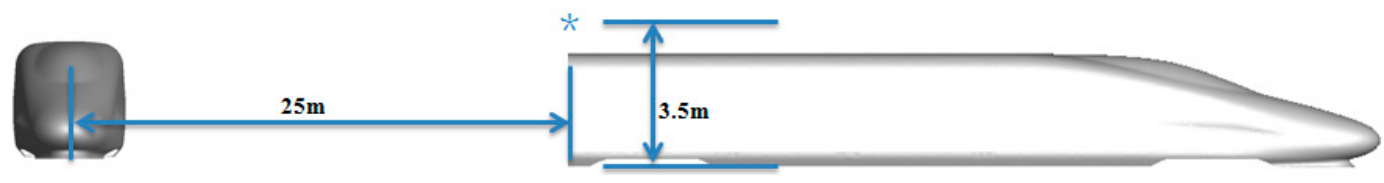

Figure 10. Schematic drawing of the observation probes in the far field.

\section{Results and Discussion}

As parameters for GA operation, the size of initial population is set to 100 . The probability of crossover is 0.9 while the probability of mutation is set to 0.3 . The size of evolution generations is set to 100 . The value of $\theta$ varies from 0 to 10 . After adding points for three times, the Kriging model meets the accuracy requirement. Figure 11 shows the convergence history of the fitness and the exact value for each variable.

The total aerodynamic drag coefficient and the equivalent continuous SPL are treated as the optimization objectives, and the multi-objective optimization of the streamlined shape has been performed on the final built Kriging model. The adaptive genetic approach with a population number of 200 is adopted. Three thousand generations have been performed in the optimization process. The roulette method is used as the selection operator, while the probabilities of crossover and mutation are set as 0.9 and 0.3 , respectively. Figure 12 shows the Pareto set of the two objectives. It can be concluded that the optimal solutions of the objectives are both limited in a small zone, indicating that specific individual in the Pareto set shows no large differences, while all the individuals are better than the initial one. In order to demonstrate the mechanism for aerodynamic performance improvement, a specific individual is chosen randomly in Figure 12 as an example, just as the red spot shows. 


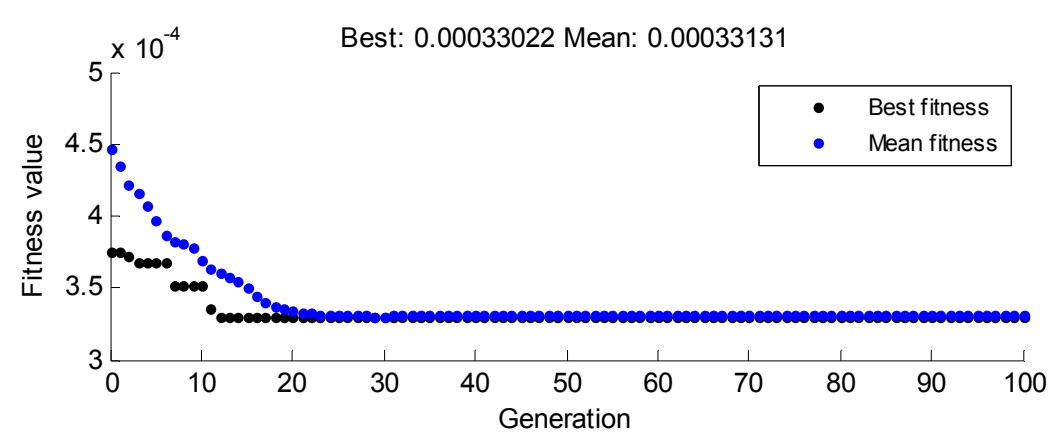

Current Best Individual

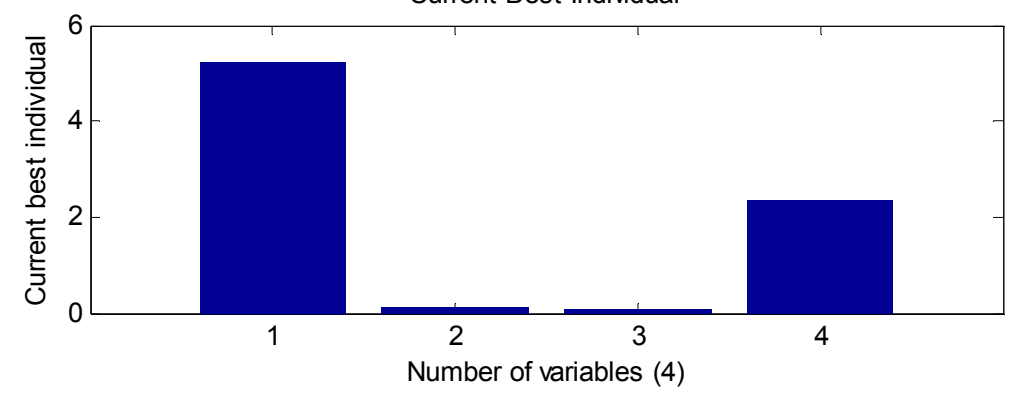

Figure 11. The convergence history of the fitness and the exact value for each variable.

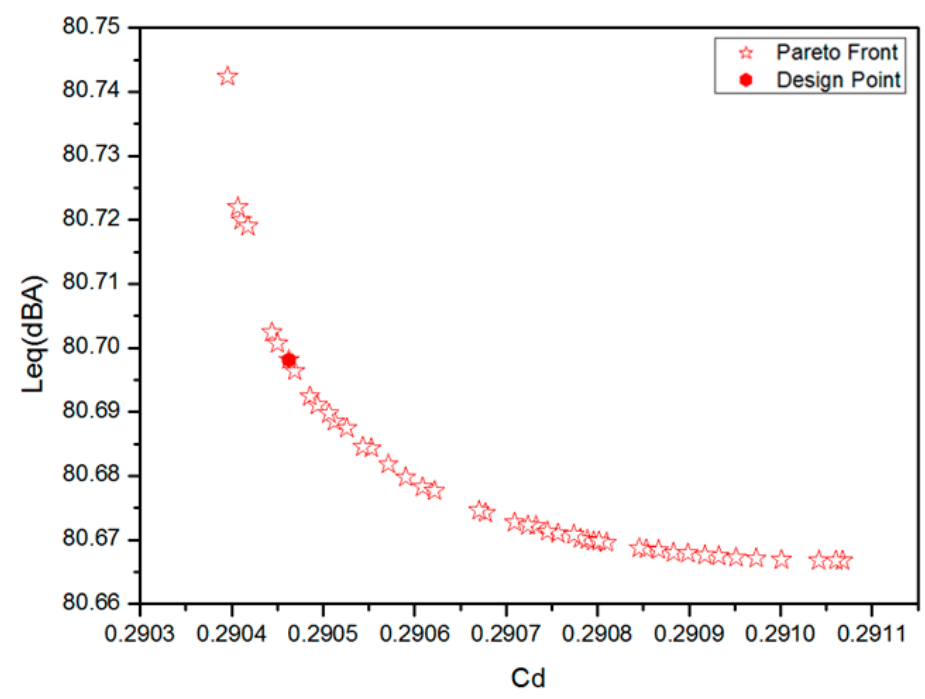

Figure 12. The Pareto front based on the drag of the whole train and the equivalent continuous SPL.

The drag coefficient of the whole train and the equivalent continuous SPL for the prototype of EMU1 are 0.314 and $81.93 \mathrm{dBA}$, respectively, while the objectives in the Pareto set are all more optimal than those of the prototype.

Table 4 shows the comparison between the CFD calculations and prediction from the Kriging model. It can be seen that excellent agreement exists between the two approaches. The errors for both objectives are $0.493 \%$ and $0.027 \%$, respectively, which can meet the engineering requirement. It can be concluded that the Kriging model built in the present paper could reveal the relationships between the design variables and the objectives. 
Table 4. The comparison between the CFD calculations and prediction from the Kriging model.

\begin{tabular}{cccccc}
\hline Objectives & Real Leq (dBA) & Kriging (dBA) & Error & Real Cd & Kriging \\
\hline Test Case & 80.676 & 80.698 & $0.027 \%$ & 0.2919 & 0.29046 \\
\hline
\end{tabular}

Figure 13 shows the variation of the streamlined shape between the prototype and the optimal result:

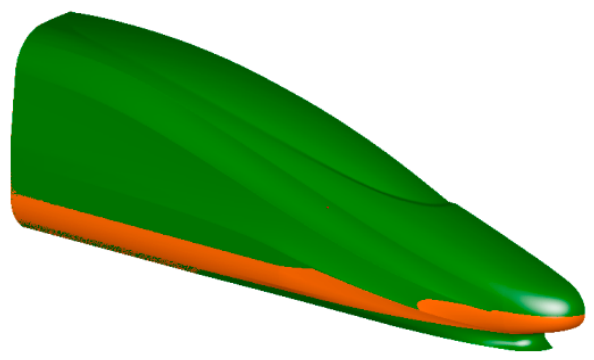

(a)

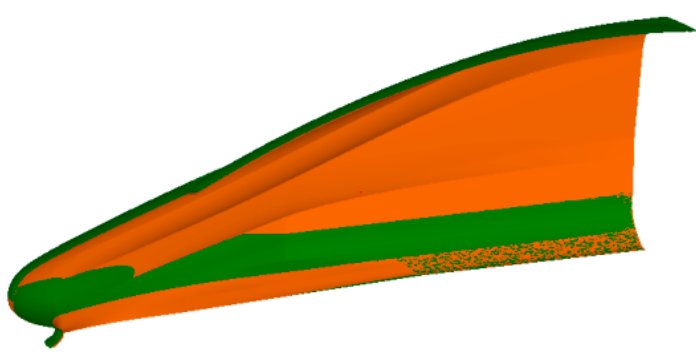

(b)

Figure 13. Comparison of the streamlined shape between the prototype and the optimal shape. (a) View from the direction of positive $y$; and (b) view from the direction of negative $y$.

As shown above, the green one represents the prototype and the orange one represents the optimal shape. Good smoothness can be observed on the shape after optimization. The width of the bottom of the streamline and the shape of the cowcatcher keep unchanged, while the width of the upper part of the streamline decreases and the heights of the nose and the cab window are both lowered.

Table 5 shows the variations of the design variables compared to the prototype model. $W_{1}$ controls the width of the streamline, $W_{2}$ controls the height of the cab window, $W_{3}$ controls the height of the nose and $W_{4}$ controls the width of the drainage. It can be seen that all the design variables get smaller.

Table 5. The variations of the design variables compared to the prototype model (1:1).

\begin{tabular}{ccccc}
\hline Variations & $W_{1} / \mathrm{mm}$ & $W_{2} / \mathrm{mm}$ & $W_{3} / \mathrm{mm}$ & $W_{4} / \mathrm{mm}$ \\
\hline Test Case & -40.85 & -38.50 & -50.81 & -61.55 \\
\hline
\end{tabular}

Table 6 shows the comparison of the objectives before and after optimization. Results reveal that the aerodynamic performance gets improved for the optimal shape. The drag coefficient and the equivalent continuous SPL are reduced by $7.1 \%$ and $1.26 \mathrm{~dB}$, respectively. For the optimization of aerodynamic drag, reducing the inviscid drag plays an important role. As seen in Table 3, the inviscid drag is reduced by $17.33 \%$.

Table 6. The comparison of the objectives before and after optimization.

\begin{tabular}{cccc}
\hline Objectives & Leq (dBA) & Cd & Cd_inviscid \\
\hline Original & 81.9316 & 0.314114 & 0.165 \\
Test Case & 80.676 & 0.2919 & 0.1364 \\
Reduction & $1.5 \%$ & $7.1 \%$ & $17.33 \%$ \\
\hline
\end{tabular}

In order to better understand the aerodynamic performance before and after optimization and investigate on the influence on the specific car by the deformation of streamlined head, the drag coefficients of each carriage before and after optimization are given in Figure 14: 


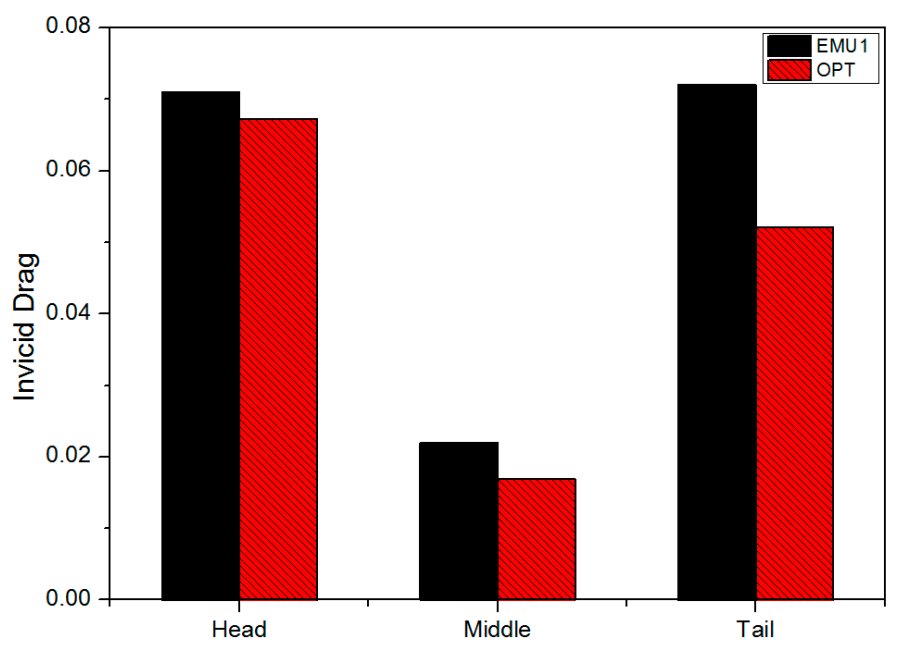

Figure 14. Comparison of drag coefficients of each carriage before and after optimization.

As shown above, the inviscid drag mainly exists on the leading car and trailing car. The inviscid drag is reduced by $5.2 \%$ and $27.6 \%$ for the leading car and trailing car, respectively. Meanwhile, the inviscid drag on the middle car is also reduced. The inviscid drag is the main source for drag optimization.

The pressure contour on the leading car before and after optimization is shown in Figure 15.

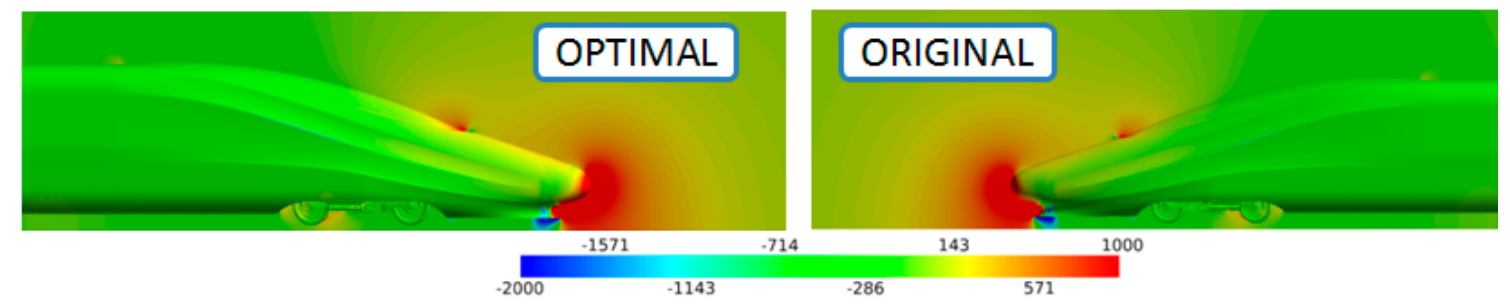

Figure 15. Pressure contour on the leading car before and after optimization (Pressure: Pa).

The height of the nose is reduced for the optimal model. Consequently, the high-pressure zone in front of the nose varies. The high-pressure zone mainly covers the region between the nose and the cowcatcher. Lower nose leads to a relatively smaller high-pressure zone, which results in a relatively smaller inviscid drag.

The pressure contour on the trailing car before and after optimization is shown in Figure 16.
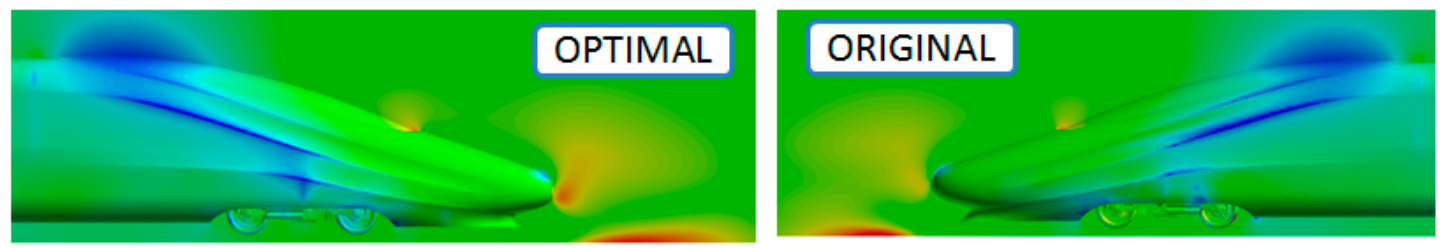

Pressure( $(\mathrm{Pa})$

$-357$

214

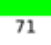

357

Figure 16. Pressure contour on the trailing car before and after optimization.

An obvious higher-pressure zone behind the trailing nose could be observed for the optimal model, which could provide a thrust on the trailing car. As a result, the inviscid drag on the trailing train could be effectively reduced. 
The pressure coefficient along the longitudinal profile of the leading car and trailing car before and after optimization is also investigated, as shown in Figure 17.
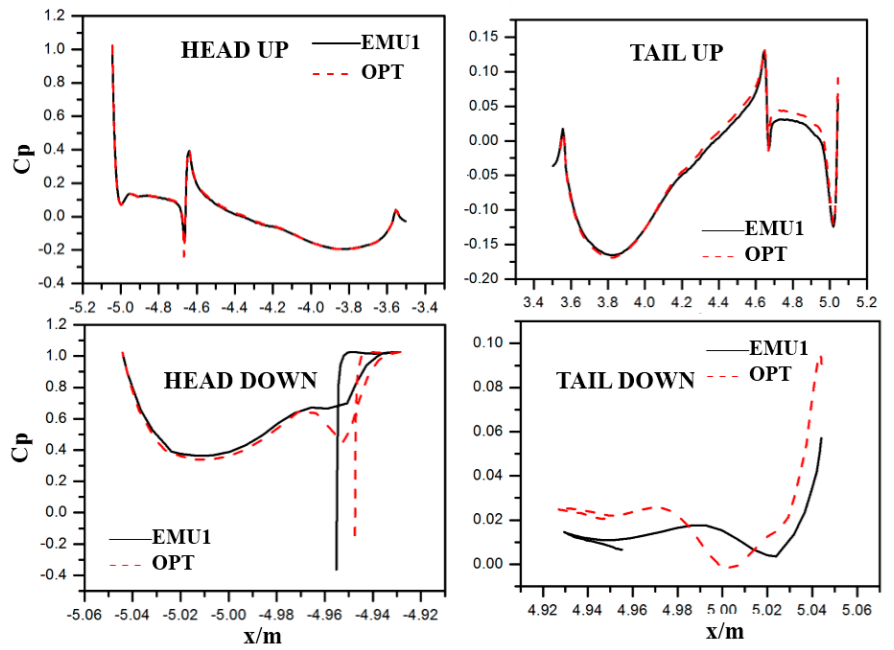

Figure 17. Pressure coefficient along the longitudinal profile of the leading car and trailing car before and after optimization.

It can be seen that the biggest variation exists at the bottom part of the trailing nose. Higher pressure could be observed along the lower longitudinal profile for the optimal model, which is the root for the relatively lower inviscid drag of the trailing car.

Then, the acoustic characteristics in the far field are analyzed. As mentioned above, the equivalent continuous SPL is reduced by $1.26 \mathrm{~dB}$. Figure 18 shows the A-weighted overall sound pressure level (OASPLA) of all the probes before and after optimization.

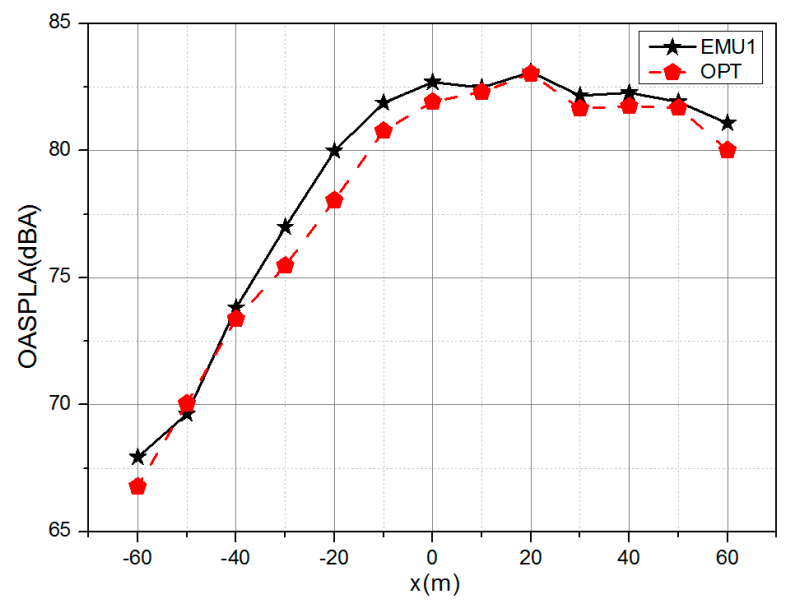

Figure 18. The Overall A-weighted Sound Pressure Levels (OASPLAs) of all the probes before and after optimization.

It can be seen that the OASPLAs of most probes for the optimal shape are reduced in a certain extent, compared to the prototype. Results reveal that the noise circumstance in the far field is improved after optimization.

In order to investigate on the influence of each design variable on the optimization objectives, it is necessary to perform the sensitivity analysis for the design variables. Sensitivity analysis is performed 
by studying the correlations between the design variables and the objectives. The correlation $r$ between variable $x$ and $y$ takes the form:

$$
r=\frac{N \sum x y-\left(\sum x\right)\left(\sum y\right)}{\sqrt{\left[\mathrm{N} \sum x^{2}-\left(\sum x\right)^{2}\right]\left[\mathrm{N} \sum y^{2}-\left(\sum y\right)^{2}\right]}}
$$

where $N$ is the number of pairs of values.

Figure 19 shows the student charts between the objectives and the design variables.

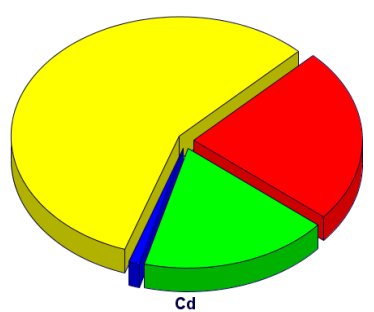

(a)

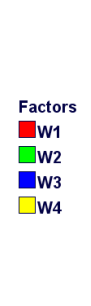

Factors
$\square$ W1
$\square$ W2
$\square$ W3
$\square$ W4

(1)

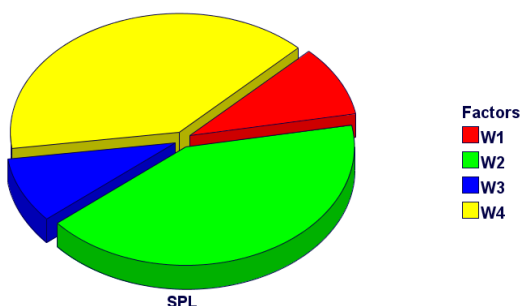

(b)

Figure 19. Student charts between the objectives and the design variables: (a) student chart between drag and four design variables; and (b) student chart between sound pressure level and four design variables.

As stated in Section 3, $W_{1}$ represents the width of the cab window, $W_{2}$ represents the height of the cab window, $W_{3}$ represents the height of the nose, and $W_{4}$ represents the width around the drainage. The design space is constrained under the real circumstance. Taking $W_{3}$ as an example, it should be not too much smaller or too much larger, or the connection between the hooks of adjacent streamlines could be affected. As seen above, $W_{4}$ has the largest influence on both the total drag and the aerodynamic noise. Besides, $W_{1}$ and $W_{2}$ own relatively large influence on the total drag and aerodynamic noise, respectively.

The three-dimensional relationship could also be established on the base of the final surrogate model. Figure 20a shows the relationship between aerodynamic noise and $W_{2}$ and $W_{4}$, while Figure 20b is the projection of aerodynamic noise on the $W_{2}-W_{4}$ plane. Strong nonlinearity could be observed. To facilitate analysis, we keep one of the design variables unchanged; the relationships of aerodynamic noise and the other design variable could be obtained, as shown in Figure 21.

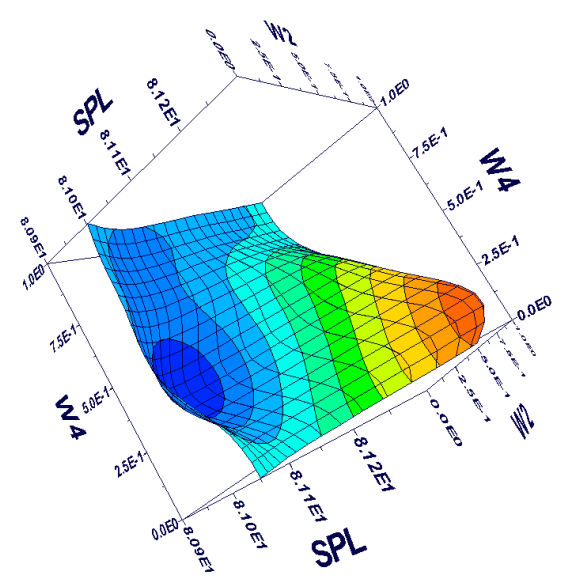

(a)

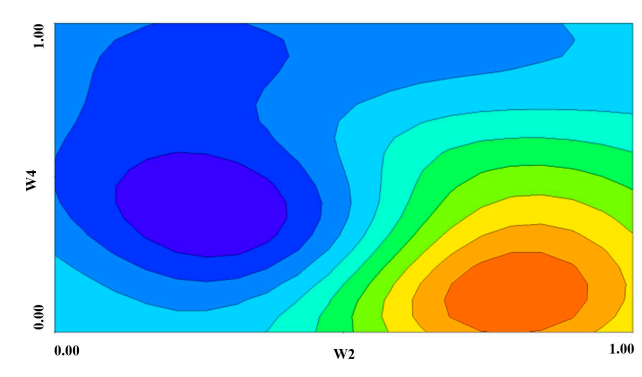

(b)

Figure 20. Relationship between aerodynamic noise and $W_{2}$ and $W_{4}$. (a) Surrogate model between aerodynamic noise and $W_{2}$ and $W_{4}$; and (b) projection of aerodynamic noise on the $W_{2}-W_{4}$ plane. 


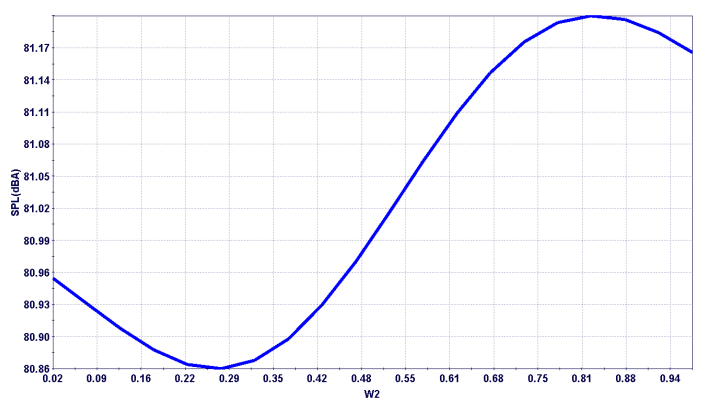

(a)

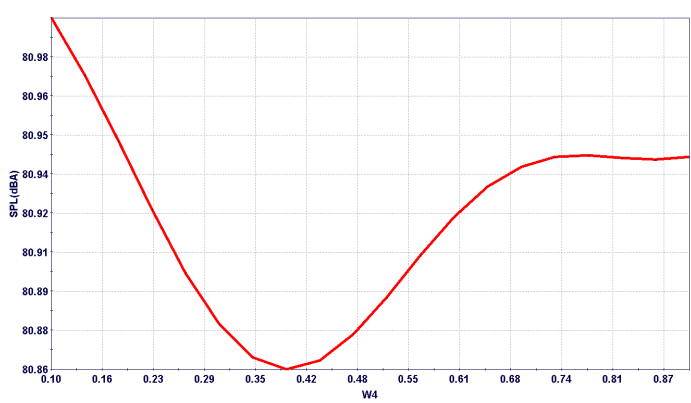

(b)

Figure 21. The relationships of aerodynamic noise and the other design variable on the condition of one design variable unchanged: (a) SPL vs. $W_{2}$; and (b) SPL vs. $W_{4}$.

Nonlinearity could also be seen in above figures, and best performance of aerodynamic noise does not appear at minimal or maximal values of $W_{2}$ or $W_{4}$. For example, it can be deduced from above figures that the width of the drainage could not be too thin or too wide, or aerodynamic noise would grow apparently.

\section{Conclusions}

An aerodynamic noise optimization strategy for high-speed trains has been proposed in the present paper. In order to take all the acoustic probes into consideration, the equivalent continuous sound pressure level (SPL) has been taken as the objective for noise optimization. In order to improve the optimization efficiency, the local shape function method has been utilized for streamlined shape parameterization, which owns stronger generality and could set design variables directly on the focused region. Meanwhile, the surrogate based optimization approach is adopted, taking the Kriging model on the base of cross-validation method as the surrogate model. Once the Kriging model is built up, multi-objective optimization by genetic algorithm could be performed on this model to avoid the computational cost of CFD/CAA for each design point, which is the key for efficiency improvement.

Taking the drag of the whole train and the equivalent continuous SPL as the objectives, a multi-objective optimization of the streamlined shape of HSTs is performed. Results reveal that the aerodynamic performance has been improved apparently compared to the prototype. The drag of the whole train has been reduced by $7.1 \%$, and the equivalent continuous SPL has been reduced by $1.26 \mathrm{~dB}$. The OASPLAs of the probes for the optimal shape are mostly reduced except for very few of them, indicating that the acoustic circumstance in the far field has been effectively improved and it is feasible to perform aerodynamic noise optimization for high-speed trains with the use of the strategy in the present paper.

Acknowledgments: This work was supported by National Natural Science Foundation of China under 11302233, and Computing Facility for Computational Mechanics Institute of Mechanics, Chinese Academy of Sciences is gratefully acknowledged.

Author Contributions: Zhenxu Sun and Guowei Yang conceived the whole numerical simulations; Ye Zhang contributed the optimization tools; and Zhenxu Sun wrote the paper.

Conflicts of Interest: The authors declare no conflict of interest.

\section{References}

1. Kalivoda, M.; Danneskiold-samsoe, U.; Kruger, F. EURailNoise: A study of European priorities and strategies for railway noise abatement. J. Sound Vib. 2003, 267, 387-396. [CrossRef]

2. Talotte, C. Aerodynamic noise: A critical survey. J. Sound Vib. 2000, 231, 549-562. [CrossRef]

3. Talotte, C.; Gautier, P.E.; Thompson, D.J.; Hanson, C. Identification, modelling and reduction potential of railway noise sources: A critical survey. J. Sound Vib. 2003, 267, 447-468. [CrossRef] 
4. Sun, Z.X.; Song, J.J.; An, Y.R. Numerical Simulation of Aerodynamic Noise Generated by High Speed Trains. Eng. Appl. Comput. Fluid Mech. 2012, 6, 173-185. [CrossRef]

5. Noger, C.; Patrat, J.C.; Peube, J. Aero-acoustical study of the TGV pantograph recess. J. Sound Vib. 2000, 231, 563-575. [CrossRef]

6. Sun, Z.X.; Guo, D.L.; Yao, S.B.; Yang, G.W.; Li, M.G. Identification and Suppression of Noise Sources around High Speed Trains. Eng. Appl. Comput. Fluid Mech. 2013, 7, 131-143. [CrossRef]

7. Ikeda, M.; Morikawa, T.; Manade, K. Development of low aerodynamic noise pantograph for high speed train. Int. Congr. Noise Control Eng. 1994, 1, 169-178.

8. Ikeda, M.; Suzuki, M.; Yoshida, K. Study on optimization of panhead shape possessing low noise and stable characteristics. Q. Rep. RTRI 2006, 47, 72-77. [CrossRef]

9. Iwamoto, K.; Higashi, A. Some consideration toward reducing aerodynamic noise on pantograph. Jpn. Railw. Eng. 1993, 122, 1-4.

10. Sueki, T.; Ikeda, M.; Takaishi, T. Aerodynamic noise reduction using porous materials and their application to high-speed pantographs. Q. Rep. Railw. Tech. Res. Inst. 2009, 50, 26-31. [CrossRef]

11. Lee, J.; Kim, J. Approximate optimization of high-speed train nose shape for reducing micropressure wave. Ind. Appl. 2008, 35, 79-87. [CrossRef]

12. Vytla, V.V.; Huang, P.G.; Penmetsa, R.C. Multi Objective Aerodynamic Shape Optimization of High Speed Train Nose Using Adaptive Surrogate Model. In Proceedings of the 28th AIAA Applied Aerodynamics Conference, Chicago, IL, USA, 28 June-1 July 2010.

13. Ku, Y.C.; Kwak, M.H.; Park, H.I.; Lee, D.H. Multi-Objective Optimization of High-Speed Train Nose Shape Using the Vehicle Modeling Function. In Proceedings of the 48th AIAA Aerospace Sciences Meeting, Orlando, FL, USA, 4-7 January 2010.

14. Yao, S.B.; Guo, D.L.; Sun, Z.X.; Yang, G.W.; Chen, D.W. Multi-objective optimization of the streamlined head of high-speed trains based on the Kriging model. Sci. China Technol. Sci. 2012, 55, 3494-3508. [CrossRef]

15. Suzuki, M.; Nakade, K. Multi-objective Design Optimization of High-speed Train Nose. J. Mech. Syst. Transp. Logist. 2013, 6, 54-64. [CrossRef]

16. Muñoz-Paniagua, J.; García, J.; Crespo, A. Genetically aerodynamic optimization of the nose shape of a high-speed train entering a tunnel. J. Wind Eng. Ind. Aerodyn. 2014, 130, 48-61. [CrossRef]

17. Batten, P.; Ribaldone, E.; Casella, M.; Chakravarthy, S. Towards a Generalized Non-Linear Acoustics Solver. In Proceedings of the 10th AIAA/CEAS Aeroacoustics Conferences, Manchester, UK, 10-12 May 2004.

18. Merci, B.; Vierendeels, J.; Langhe, C.D.; Dick, E. Development and Application of a New Cubic Low-Reynolds Eddy-Viscosity Turbulence Model. In Proceedings of the 31st AIAA Fluid Dynamics Conference \& Exhibit, Anaheim, CA, USA, 11-14 June 2001.

19. Williams, J.E.F.; Hawkings, D.L. Sound generation by turbulence and surfaces in arbitrary motion. Philos. Trans. R. Soc. Lond. Ser. A Math. Phys. Sci. 1969, 264, 321-342. [CrossRef]

20. Casper, J.; Farassat, F. A new time domain formulation for broadband noise predictions. Int. J. Aeroacoustics 2002, 1, 207-240. [CrossRef]

21. Lee, I.; Sung, H.J. Characteristics of wall pressure fluctuations in separated and reattaching flows over a backward-facing step: Part 1. Time-mean statistics and cross-spectral analyses. Exp. Fluids 2001, 30, 262-272. [CrossRef]

22. Blazek, J. Computational Fluid Dynamics Principles and Applications; Elsevier Ltd.: Amsterdam, The Netherlands, 2005; pp. 16-17.

23. Menter, F.R. Zonal Two Equation K-W Turbulence Models for Aerodynamic Flows. In Proceedings of the 24th AIAA Fluid Dynamics Conference, Orlando, FL, USA, 6-9 July 1993.

24. Sederberg, T.W.; Parry, S.R. Free-Form deformation of solid geometric models. SIGGRAPH Comput. Graph. 1986, 20, 151-160. [CrossRef]

25. Piegl, L.; Tiller, W. The NURBS Book. In Monographs in Visual Communication; Springer: Berlin/Heidelberg, Germany, 1997.

26. Noel, C. The Origins of Kriging. Math. Geol. 1990, 22, 239-252.

27. Donald, R.J.; Matthias, S.; William, J.W. Efficient Global Optimization of Expensive Black-Box Functions. J. Glob. Optim. 1998, 13, 455-492.

28. Donald, R.; Jones, A. Taxonomy of Global Optimization Methods Based on Response Surfaces. J. Glob. Optim. 2001, 21, 345-383. 
29. Gao, Y.H.; Wang, X.C. An effective warpage optimization method in injection molding based on Kriging model. Int. J. Adv. Manuf. Technol. 2008, 37, 953-960. [CrossRef]

30. Markatou, M.; Biswas, S. Analysis of variance of cross-validation estimators of the generalization error. J. Mach. Learn. Res. 2005, 6, 1127-1168. 